\title{
Repercussões do fechamento da Unidade de Desintoxicação do Hospital Psiquiátrico São Pedro
}

\author{
Renata Brasil Araujo* \\ Luisa Isabel Dufech Gimeno** \\ Rita Mello de Mello*** \\ Elaine Brasil Ruschel $\left.\right|^{* * * *}$ \\ Lucas S. Benevides*** \\ Roberto C. Nichetti***
}

\section{INTRODUÇÃO}

De acordo com Araujo et al. ${ }^{1}$, o tratamento específico para dependência química no Hospital Psiquiátrico São Pedro iniciou na década de 40 com uma Unidade de Internação denominada "Pavilhão de Alcoolistas". Esta Unidade foi a primeira ação no sentido de tratar o dependente químico respeitando suas peculiaridades, não o internando com pacientes de outras psicopatologias.

A Unidade de Desintoxicação Jurandy Barcellos, segundo Araujo et al. ${ }^{1}$, foi a última ala de

Instituição: Hospital Psiquiátrico São Pedro -Ambulatório Melanie Klein Instituição onde o trabalho foi realizado: Hospital Psiquiátrico São Pedro Serviço da Admissão e Triagem.

Endereço: Av Bento Gonçalves, 2460 - Bairro Partenon- Porto Alegre - Rio Grande do Sul.

Trabalho não subvencionado.

* Psicóloga do Ambulatório Melanie Klein/HPSP, Mestre em Psicologia Clínica, Doutoranda em Psicologia.

** Psiquiatra do Ambulatório Melanie Klein/HPSP.

*** Residente de Enfermagem do HPSP.

${ }^{* * * *}$ Residente de Terapia Ocupacional do HPSP.

${ }^{* * * * *}$ Residentes de Psiquiatria do HPSP. internação pacientes do sexo masculino de Porto Alegre e interior do Estado do Rio Grande do Sul, com dependência de substâncias psicoativas. O seu tempo médio de internação era de 7 a 15 dias e, além de ser realizada a desintoxicação, havia um tratamento tendo como referencial a Entrevista Motivacional ${ }^{2}$ e o modelo da Prevenção de Recaída ${ }^{3}$, que fazem parte da Terapia Cognitivo-Comportamental, que, conforme Oliveira ${ }^{4}$, é a mais adequada no tratamento de pacientes com esta psicopatologia.

Os pacientes com diagnóstico de Transtorno Psicótico Induzido por Substância ou com possível comorbidade primária, na qual havia sintomas psicóticos, a princípio, eram internados em outras Unidades hospitalares específicas: Unidade Mário Martins Masculina e José de Barros Falcão. Na Unidade Jurandy Barcellos, eram atendidos em média 40 pacientes por mês. ${ }^{1}$

No dia 6 de agosto de 2002, tendo como fundamento a Lei da Reforma Psiquiátrica do Deputado Paulo Delgado 5 , na qual é previsto o progressivo fechamento de leitos em hospitais psiquiátricos e a substituição destes por leitos em hospitais gerais, foram proibidas novas in- 
ternações na Unidade Jurandy Barcellos, havendo uma transferência dessas vagas para o Hospital Vila Nova a partir de um convênio firmado entre a Secretaria Estadual da Saúde e a Prefeitura Municipal de Porto Alegre.

Os pacientes que já estavam internados na Unidade do Hospital São Pedro ficaram até a sua alta hospitalar, sendo que o fechamento definitivo da Unidade Jurandy Barcellos com a saída do último paciente ocorreu no dia 11 de setembro de 2002.

Percebendo o quanto ainda são carentes as vagas para atendimento gratuito de dependentes químicos, não só no Rio Grande do Sul como em todo o Brasil, bem como preocupado com o descumprimento da Legislação do Sistema Único de Saúde ${ }^{6}$, o Sindicato Médico do Rio Grande do Sul juntamente com a Sociedade de Apoio ao Doente Mental (SADOM) mobilizaram forças para que estes leitos fossem reabertos.

De acordo com os levantamentos estatísticos realizados pelo Serviço de Admissão e Triagem do Hospital Psiquiátrico São Pedro, neste período, houve um aumento de pacientes dependentes químicos aguardando vaga para internação, alguns tendo de ficar até mais de 24 horas em serviço de emergência e, por outro lado, deu-se um aumento no número de ordens judiciais determinando a internação de dependentes químicos em outras unidades do Hospital Psiquiátrico São Pedro.

O movimento de reforma psiquiátrica, definido como a articulação política de renovação da estrutura de funcionamento dos serviços de atenção à saúde mental, difunde-se pelo mundo através de programas governamentais amparados por lei. A literatura médica internacional está repleta de descrições do processo de reforma psiquiátrica em vários países, destacando-se a Itália ${ }^{7}$, Espanha ${ }^{8,9}$, Rússia $^{10}$, Alemanha ${ }^{11}$, Grécia ${ }^{12}$ e o Brasil, na cidade de São Lourenço do Sul, Rio Grande do Sul ${ }^{13}$. Em cada um destes países e em outros, o processo de reforma encontra-se em diferentes etapas, e cada país enfrenta empecilhos diferentes para a sua implementação.

Rotelli ${ }^{14}$, ao discorrer sobre o processo de desinstitucionalização na Itália, faz um paralelo com a experiência norte-americana, apontando para o fato de que o insucesso do processo de desinstitucionalização nos Estados Unidos decorreu do fato de que o processo reduziu-se à desospitalização. Uma política apressada de esvaziamento de hospitais não ocorreu concomitante ao reforço e implementação de outros serviços substitutivos à internação, o que gerou uma enorme demanda desassistida, e o fim de um grande problema tornou-se o início de um problema maior: a geração de uma massa marginalizada e privada de assistência.

Os objetivos e metas da reforma psiquiátrica nos países europeus onde o processo de reforma ganhou dimensão mais significante são: o desenvolvimento de uma nova organização de um cuidado em saúde mental, de caráter descentralizado e com base territorial; integração dos pacientes psiquiátricos nos cuidados de saúde primária; a criação de uma extensa comunidade de centros de saúde; desenvolvimento de atitudes mais positivas acerca do adoecer mental ${ }^{8}$. Além destes, são citados: proibição de admissão de pacientes em hospitais mentais estatais, estipulação de serviços de comunidade, hospitalização apenas em unidades pequenas em hospital geral e criação de serviços comunitários e residenciais ${ }^{7,11}$. As estratégias terapêuticas empregadas por novos serviços em saúde mental incluem: promover a permanência dos pacientes perto de suas esferas familiar e social, atenção integral às necessidades individuais, respeito às diferenças individuais, práticas reabilitativas e reinserção social $^{13}$.

O Rio Grande do Sul tornou-se pioneiro na reforma psiquiátrica brasileira, o que se reflete na aprovação da primeira lei de Reforma Psiquiátrica (lei estadual número 9716, de 07 de agosto de 1992) do Brasil. A lei da reforma psiquiátrica dispõe sobre a reforma psiquiátrica no Rio Grande do Sul, determina a substituição progressiva dos leitos nos hospitais psiquiátricos por rede de atenção integral em saúde mental, determinada por regras de proteção aos que padecem de sofrimento psíquico, especialmente quanto às internações psiquiátricas compulsórias, e dá outras providências ${ }^{5}$. No seu artigo 2, a lei cita que a reforma psiquiátrica consistirá na gradativa substituição do sistema hospitalocênctrico de cuidados às pessoas que padecem de sofrimento psíquico por uma rede integrada e por variados serviços assistenciais de atenção sanitária e social, tais como ambulatórios, emergências psiquiátricas em hospitais gerais, leitos ou unidades de internação psiquiátrica em hospitais gerais, hospitais-dia, hospitais-noite, centros de convivência, centros comunitários, centros de atenção psicossocial, centros residenciais de cuidados intensivos, lares abrigados, pensões públicas comunitárias, oficinas de atividades construtivas e similares. No seu artigo 5 , a lei cita que, quando da construção de hospitais gerais no Estado, será requisito imprescindível a existência de serviço de atendimento para pacientes 
que padeçam de sofrimento psíquico, guardadas as necessidades de leitos psiquiátricos locais e/ou regionais ${ }^{5}$.

No que diz respeito ao tratamento à assistência à saúde de dependentes químicos, as necessidades de leitos, que devem ser guardadas de acordo com as demandas locais e regionais, obrigam os programas de reforma psiquiátrica a contemplarem a crescente demanda por atenção à saúde do dependente químico, o que decorre do aumento da incidência de casos em nosso meio ${ }^{15}$.

\section{OBJETIVOS}

Medir se houve aumento na prevalência de pacientes dependentes químicos internados nas unidades de psicóticos adultos com o fechamento da unidade de desintoxicação Jurandy Barcellos e medir se houve aumento no número de dependentes químicos para os quais tenha sido recusada vaga para internação.

\section{MÉTODO}

Primeiramente, foi elaborado um projeto de pesquisa que foi avaliado e aprovado pelo Comitê de Ética do Hospital Psiquiátrico São Pedro.

A amostra foi composta por dois sub-grupos: 1) todos os pacientes internados nas Unidades Mário Martins Masculina e José de Barros Falcão, do Hospital Psiquiátrico (ambas unidades de internação que atendem pacientes adultos e, em sua maioria, psicóticos) nos três meses anteriores ao fechamento da Unidade de Desintoxicação Jurandy Barcellos (período de 06/05/2002 a 06/08/2002); 2) todos os pacientes internados nas Unidades Mário Martins Masculina e José de Barros Falcão, do Hospital Psiquiátrico nos três meses posteriores ao fechamento da Unidade de Desintoxicação Jurandy Barcellos (período de 06/08/2002 a 06/ 11/2002).

Além disto, foi analisado o número de dependentes químicos para os quais foram recusadas vagas para internação, devido à falta de leitos ou pela incoerência de sua hospitalização em uma Unidade psiquiátrica não especializada em dependência química (06/05/2002 a 06/11/2002).

Foi realizado um levantamento retrospectivo de dados no livro-registro de internações do Serviço de Admissão e Triagem do Hospital Psiquiátrico São Pedro, bem como dos prontuários dos pacientes, o que constitui uma amostra não aleatória ou por conveniência.
Os dados foram tratados estatisticamente, sendo feita uma análise descritiva dos dados sócio-demográficos, utilizando-se do Teste T de Student para a comparação das médias entre os grupos e o Teste Qui-Quadrado para avaliar a associação entre variáveis categóricas. O nível de significância foi de 5\%.

\section{RESULTADOS}

Foram analisados um total de 261 sujeitos, sendo que 128 (49\%) internaram na Unidade Mário Martins Masculina e 133 (51\%), na Unidade José de Barros Falcão. A média de idade $(n=261)$ foi de $31,58(d p=12,58 ; 13-75)$, sendo a média de idade antes do fechamento equivalente a $31,44(d p=12,47)$ e depois do fechamento igual a $31,73(d p=12,75)$. De acordo com o Teste T para comparação entre duas amostras independentes, não houve diferença significativa entre os dois grupos $(p=0,851)$ quanto à idade.

Quanto ao nível de escolaridade, a média foi de 5,3 anos de estudos $(\mathrm{dp}=3,16 ; 0-15)$ no total da amostra $(n=261)$. A média de anos de estudo antes do fechamento foi de 5,69 $(\mathrm{dp}=3,30)$ e, depois do fechamento, igual a 4,99 $(\mathrm{dp}=3,03)$. O tempo médio de internação antes do fechamento foi de 29,71 dias $(d p=20,04)$ e, depois do fechamento, igual a 30,39 dias $(d p=14,22)$. Segundo o Teste T para comparação entre duas amostras independentes, não houve diferença significativa entre os dois grupos quanto à escolaridade $(p=0,134)$ nem quanto ao tempo médio de internação $(p=0,752)$.

Quanto à procedência, 28 sujeitos (10,7\%) eram de Porto Alegre, 115 (44,1\%) da Grande Porto Alegre e 118 (45,2\%) do Interior do Rio Grande do Sul ( $n=261)$.

Antes do fechamento, foram atendidas 13 ordens judiciais $(9,6 \%)$ e depois do fechamento $19(15,1 \%)$, não havendo diferença significativa entre estes dois grupos pelo Teste QuiQuadrado $\left(X^{2}=1,80 ; p=0,179\right)$.

Ao se comparar o número de pacientes que tinham diagnóstico de dependência química (independente de ter outra comorbidade psiquiátrica) constatou-se que 21 sujeitos (15,6\%) com este perfil internaram antes do fechamento e 28 $(22,2 \%)$ depois. Com relação ao diagnóstico de surto psicótico induzido por substâncias psicoativas, $6(4,4 \%)$ internaram antes e 11 (8,7\%) depois do fechamento. Não foi encontrada diferença significativa entre os dois grupos quanto à presença do diagnóstico de dependência química $\left(X^{2}=1,90 ; p=0,167\right)$, nem quanto à ocorrência de surtos psicóticos induzidos por subs- 
tâncias psicoativas $\left(X^{2}=1,98 ; p=0,158\right)$.

A distribuição dos diagnósticos principais dos pacientes internados $(n=261)$ é demonstrada na Tabela 1, e os diagnósticos associados à dependência de alguma substância psicoativa $(n=261)$ são apresentados na Tabela 2, ambas em anexo.

Tabela 1. Distribuição dos Diagnósticos Principais dos pacientes internados $(n=261)$. Porto Alegre, 2003.

\begin{tabular}{lrr}
\hline Diagnóstico & $\mathrm{N}$ & $\%$ \\
\hline Dependência Química & 41 & 15,7 \\
Transtornos do tipo Esquizofrênicos & 100 & 38,3 \\
Transtornos de Humor & 72 & 27,6 \\
Transtornos de Ansiedade & 03 & 1,1 \\
Transtornos de Personalidade & 10 & 3,8 \\
Retardo Mental & 13 & 5,0 \\
Transtornos Orgânicos & 18 & 6,9 \\
Outros & 04 & 1,5 \\
Total & 261 & 100 \\
\hline & &
\end{tabular}

Tabela 2. Distribuição dos Diagnósticos referentes à Dependência Química $(n=261)$. Porto Alegre, 2003.

\begin{tabular}{lrr}
\hline Diagnóstico & $\mathrm{n}$ & $\%$ \\
\hline Nenhum & 212 & 81,2 \\
Dependência de álcool & 21 & 8,0 \\
Dependência de cannabis & 01 & 0,4 \\
Dependência de cocaína & 02 & 0,8 \\
Dependência de benzodiazepínicos & 01 & 0,4 \\
Dependência de Solventes & 03 & 1,1 \\
Dependência de Múltiplas Substâncias & 21 & 8,0 \\
Total & 261 & 100 \\
\hline
\end{tabular}

Quando analisado o número de pacientes com somente o diagnóstico de dependência química (sem comorbidades psiquiátricas) internados, observou-se que, antes do fechamento, internaram 15 sujeitos $(11,1 \%)$ e depois 26 $(20,6 \%)$, o que é, em termos de diferença, estatisticamente significativo $\left(X^{2}=4,497 ; p=0,033\right)$. Se excluirmos deste grupo de pacientes com diagnóstico único aqueles que apresentaram um surto psicótico induzido pela substância psicoativa, obtemos 9 sujeitos que internaram antes do fechamento $(6,7 \%)$ e 15 depois $(11,9)$. Neste último caso, não há diferença significante entre os dois grupos ( $\left.X^{2}=2,15 ; p=0,142\right)$.

Foi realizada uma comparação da recusa de vagas para internação realizada no Serviço de Admissão e Triagem do Hospital Psiquiátrico São Pedro antes e depois do fechamento da Unidade Jurandy Barcellos (3 meses antes e 3 meses depois), e obteve-se que o número de recusas antes do fechamento foi de 32 (24 destes internaram em outro hospital) e depois, de 168 , dos quais somente 10 internaram em outro hospital.

Foi aplicado o Teste Qui-Quadrado para comparar o número de recusas de vagas para internação antes e depois do fechamento da Unidade Jurandy Barcellos e o resultado foi bastante significativo $\left(X^{2}=154,40 ; p=0,0000\right)$ : houve um aumento substancial no número de pacientes que ficaram sem leito hospitalar.

\section{DISCUSSÃO DOS RESULTADOS}

A partir da análise dos resultados obtidos, podemos observar que, após o fechamento da Unidade Jurandy Barcellos, não houve um aumento significativo quanto à internação de dependentes químicos nas unidades psiquiátricas que atendem a pacientes psicóticos $\left(X^{2}=1,90\right.$; $p=0,167)$. Houve, no entanto, um aumento considerável de pacientes que eram dependentes químicos, não apresentavam comorbidades psiquiátricas e internaram em unidades de psicóti$\cos \left(X^{2}=4,497 ; p=0,033\right)$, não existindo uma possibilidade de se oferecer um atendimento especializado para dependência química. No entanto, é preciso destacar que alguns destes pacientes apresentavam um surto psicótico induzido, o que torna a indicação para as unidades Mário Martins Masculina e José de Barros Falcão adequada.

Como não houve um aumento global no número de dependentes químicos internados em unidades de psicóticos, após o fechamento da Unidade Jurandy Barcellos, a pergunta que se faz é quanto ao destino que estes pacientes tiveram. A resposta, neste caso, é bastante desapontadora: 168 pacientes não foram internados no Hospital Psiquiátrico São Pedro e apenas 10 internaram em outros hospitais, havendo um aumento significativo no número de pacientes que ficaram sem assistência $\left(X^{2}=154,40 ; p=0,0000\right)$.

Sabemos que o número de serviços de atendimento especializado em dependência química pelo SUS, tanto em termos de internação quanto nos Postos de Saúde, é pequeno e que a prevalência de pacientes com este diagnóstico tem aumentado a cada $\mathrm{ano}^{15}$. Assim, apesar de estarmos de acordo com o que preconiza a Reforma Psiquiátrica, quanto à substituição dos leitos em hospitais psiquiátricos por 
leitos em hospitais gerais ${ }^{(5)}$, consideramos que, em primeiro lugar, é necessário que todos os indivíduos tenham acesso aos serviços de saúde, conforme a Legislação do SUS ${ }^{6}$, não havendo nenhum caso de recusa a atendimento e sendo ampliados os serviços substitutivos e de atenção primária ${ }^{5,7,8,11,13,14}$.

É importante que as experiências já realizadas em outros países e até mesmo no Brasil quanto à Reforma Psiquiátrica ${ }^{7-14}$ sejam utilizadas como parâmetros para que sejam delineadas as estratégias na área de saúde mental em nosso país.

A Reforma Psiquiátrica deve sim ser uma meta a ser seguida por todos os profissionais de saúde; no entanto, não podemos deixar de lado as necessidades básicas e específicas de nossos pacientes ${ }^{2-4}$ para que não sejamos guiados por uma lógica perversa, que desconsidera aspectos técnicos ${ }^{14}$.

\section{CONCLUSÕES}

O fechamento de uma unidade de internação para dependentes químicos em um hospital psiquiátrico está previsto na lei estadual de reforma psiquiátrica. $\mathrm{O}$ fechamento deste tipo de serviço, segundo a própria lei de reforma psiquiátrica e segundo toda uma construção teórico-política feita pelo movimento antimanicomial, prevê uma lenta e gradual substituição de leitos psiquiátricos de hospital psiquiátrico por leitos psiquiátricos em hospital geral, além da construção concomitante de serviços substitutivos à internação psiquiátrica, quando esta pode ser substituída ${ }^{5}$.

Os achados de nosso estudo demonstraram que uma pequena parte da demanda de dependentes químicos necessitando de uma internação foi apenas deslocada para a internação em unidades de psicóticos em hospital psiquiátrico, impondo aos portadores, tanto de psicoses como de dependência química, um ônus terapêutico, já que o tratamento de desintoxicação e reabilitação de dependentes químicos, que idealmente deve ser realizado em hospital geral ou ambulatorialmente, caso não haja riscos clínicos, passa a ocorrer em unidades de psicóticos, o que se constitui em um contrasenso terapêutico.

Demonstrou-se ainda que grande parte desta clientela ficou sem assistência especializada em dependência química, recebendo um primeiro atendimento de emergência, mas sendo recusada a vaga para internação hospitalar, devido ao número insuficiente de leitos disponíveis pelo SUS.
Como todos os estudos, este tem uma limitação quanto ao número de sujeitos, pois apesar do total da amostra de pacientes internados ser equivalente a 261, somente 15 (antes do fechamento) e 26 (depois do fechamento) eram dependentes químicos. Desta forma, este aumento de 11 indivíduos, em termos estatísticos, não pode ser considerado significativo, o que provavelmente o seria caso a amostra fosse maior.

No entanto, o que se pretende destacar é que a implementação da reforma psiquiátrica, quando efetivada sem o devido planejamento e sem respeitar as particularidades de cada contexto e as limitações com relação à capacidade da rede de atender à demanda de problemas, acaba por acarretar ônus para os que sofrem com a dependência química: termina por repetir um processo de desospitalização, cujo resultado é a desassistência.

Quando nos referimos aos dependentes químicos, devemos ter ainda um cuidado complementar, à medida que estes sujeitos já sofrem vários tipos de discriminação em seu dia-a dia. Se é estigmatizante internar em um hospital psiquiátrico, não podemos deixar de pensar que é muito mais ser colocado à margem do Sistema de Saúde, o que pode, inclusive, acarretar riscos de vida e, na melhor das hipóteses, diminuir as chances de recuperação.

\section{REFERÊNCIAS BIBLIOGRÁFICAS}

1. Araujo RB, Souto KEP, Gimeno LID, Athayde LV, Souza ACF, Lopes SR \& Capra NV. Perfil de Pacientes Dependentes Químicos Atendidos no Hospital Psiquiátrico São Pedro-RS. Revista de Psiquiatria do Rio Grande do Sul, v. 24, no 2,maio/agosto, 2002: p.143-150

2. Miller W \& Rollnick S. Entrevista Motivacional: preparando as pessoas para a mudança de comportamentos adictivos. Porto Alegre: Artes Médicas, 2001.

3. Marlatt A \& Gordon J. Prevenção de Recaída - Estratégias de Manutenção no Tratamento de Comportamentos Adictivos. Porto Alegre: Artes Médicas, 1993.

4. Oliveira M. Entrevista Motivacional. In Cunha, Jurema e Colaboradores. Psicodiagnóstico V. (p.p. 88-95). Porto Alegre: Artes Médicas, 2000.

5. Diário Oficial do Rio Grande do Sul. Lei estadual número 9716, de 07 de agosto de 1992, Rio Grande do Sul, 1992.

6. Brasil. Leis e Decretos. Lei 8080 de 19 de setembro de 1990, Brasília, Ministério da Saúde, 1990.

7. Burti L. Italian, psychiatric reform 20 plus years after. Acta Psych Scan 2001; 104, supl. 410, 41-46.

8. Vazquez BJL, Garcia J, Gonzales TF. Spanish psychiatric reform: what can be learned from two decades of experience? Acta Psych Scan 2001; 104, suppl. 410, 8995

9. Basauri A, Victor, Gutierres S, Esther A. Psychiatric reform in Spain. Int J Mental Health, 2002 fal; vol 31 (3): 1429. 
10. Poloshij B, Saposhnikova I. Psychiatric reform in Russia. Acta Psych Scan 20012; 104, suppl. 410, 56-62.

11. Bauer M, Kunze H, Von Cranach M, Fritze J, Becker T. Psychiatric reform in Germany. Acta Psych Scan 2001; 104, suppl 410, 27-34

12. Madianos MG. Desinstitutionalization and the closure of public mental hospitals: the pragmatics of psychiatric reform in Greece. International Journal of Mental Health. 2002 fal; vol 31(3): 66-75.

13. Hirdes A, Kantorski LP. Care systematization is psychiatric nursing witin the psychiatric reform context. Journal of Psychiatric and Mental Health Nursing 2002 feb; vol. 9 (1): $81-86$.

14. Rotelli F et. al. Desinstitucionalização. São Paulo, Editora Hucitec, 1990.

15. Carlini, E. A. et al. I Levantamento domiciliar sobre o uso de drogas psicotrópicas no Brasil: estudo envolvendo as 107 maiores cidades do país. São Paulo: CEBRID: UNIFESP, 2002.

\section{RESUMO}

Introdução: $A$ lei de reforma psiquiátrica prevê substituição de leitos em hospitais psiquiátricos por leitos em hospitais gerais, e o fechamento da unidade de desintoxicação do Hospital Psiquiátrico São Pedro está de acordo com estes princípios.

Objetivos: Mensurar se aumentou a prevalência de dependentes químicos internados em unidades de psicóticos deste hospital antes e depois do fechamento da unidade de desintoxicação e se aumentaram as recusas de vagas para internação.

Método: Estudo transversal, com amostra não aleatória, dividida em: Grupo 1, com todos os pacientes internados em unidades que atendem adultos masculinos três meses antes do fechamento e Grupo 2, com todos os internados, nestas unidades, nos três meses posteriores. Analisou-se o número de vagas recusadas para dependentes químicos. Realizou-se um levantamento retrospectivo em registros e prontuários.

Resultados: A amostra foi de 135 do Grupo 1 e 126 do Grupo $2(n=261)$. No Grupo 1, 15,6 \% tinham o diagnóstico de dependência química e, no Grupo 2, $22,2 \%$. Aumentaram as internações de dependentes químicos sem co-morbidades psiquiátricas $\left(X^{2}=4,497\right.$; $p=0,033)$ e o número dos que ficaram sem vaga $\left(X^{2}=154,40 ; p=0,0000\right)$.

Discussão: Após o fechamento, houve um aumento de dependentes químicos, sem co-morbidades psiquiátricas que internaram em unidades de psicóticos, sendo privados de atendimento especializado. Também se observou um aumento de desassistidos, o que fere a reforma psiquiátrica.

Conclusão: A Reforma Psiquiátrica deve ser uma meta a ser seguida pelos profissionais da saúde; no entanto, não se pode esquecer das necessidades específicas dos pacientes nem desconsiderar os aspectos técnicos.

Descritores: Dependência Química, reforma psiquiátrica, desinstitucionalização.

\section{ABSTRACT}

Introduction: The psychiatric care reform law foresees replacing psychiatric hospital beds by general hospital beds, and closing the Hospital Psiquiátrico São Pedro detoxification unit complies with these principles.

Objectives: To evaluate whether the number of chemically-dependent patients admitted to the psychotics units at this hospital increased before and after the detoxification unit was closed, and whether the availability of hospital beds has decreased.

Method: a cross-sectional study with a nonrandom sample was used. It was divided into: Group 1 , with all patients admitted to units caring for adult males three months before closure and Group 2, all patients admitted to these units in the three months after that unit was closed. The number of places refused to chemical dependents was analyzed, and a retrospective assessment of records and medical files was performed.

Results: The sample in Group 1 was 135, while Group 2 was $126(n=261)$. In Group 1, 15.6\% were diagnosed as being chemically dependent and, in Group 2, 22.2\%. There was an increase in the number of chemically-dependent patients admitted without psychiatric comorbidities $\left(X^{2}=4,497 ; p=0,033\right)$ and in the number of those who could not be admitted ( $X^{2}$ $=154,40 ; p=0,0000$ ).

Discussion: Since the unit was closed, there has been an increase in the number of chemically addicted patients, without psychiatric comorbidities who were hospitalized in units for psychotics, and deprived from specialized treatment. It has also been noticed that there has been an increase in patients who do not receive treatment, which goes against the intention of the psychiatric care reform.

Conclusion: Psychiatric Care Reform should be an objective to be pursued by all health professionals. However, we must neither overlook the patients' special needs nor neglect technical aspects.

Keywords: Chemical Dependence, psychiatric care reform, deinstitutionalization.

Title: Repercussions of closing the Detoxification Unit at Hospital Psiquiátrico São Pedro

\section{RESUMEN}

Introducción: La ley de reforma psiquiátrica prevé sustituir lechos en hospitales psiquiátricos por lechos en hospitales generales e el cierre de la unidad de desintoxicación del Hospital Psiquiátrico San Pedro esta de acuerdo con esos principios.

Objetivos: Evaluar si aumentó la prevalencia de dependientes químicos internados en unidades psiquiátricas de este hospital antes y después del cierre 
de la unidad de desintoxicación y si aumentasen casos de rechazo de una vacante para la internación.

Método: Estudio transversal, con una muestra no aleatoria, dividida en: Grupo1, con todos los pacientes internados en unidades que atienden adultos varones tres meses antes del cerramiento y Grupo 2 con todos los internados, en estas unidades, en los tres meses después. Fue hecha una análisis en el número de vacantes rechazadas para dependientes químicos. También fue realizado un levantamiento retrospectivo en registros y prontuarios.

Resultados: La muestra fue de $135 \mathrm{del}$ grupo $1 \mathrm{e}$ 126 del grupo 2 ( $n=261)$. En el grupo 1, 15,6\% tenían el diagnóstico de dependencia química y, en el grupo 2, 22,2\%. Aumentaron las internaciones de dependientes químicos sin diagnóstico dual psiquiátrico. $\left(x^{2}=\right.$ 4,497; $p=0,033)$ e el número de los que quedaron $\sin$ vacante. $\left(x^{2}=154,40 ; p=0,000\right)$

Discusión: Después del cierre, ocurrió un aumento de dependientes químicos, sin co-morbidade psiquiátrica que internaron en unidades de psicóticos, no recibiendo atendimiento especializado. Se observó también un aumento de pacientes no asisti- dos, lo que hiere la reforma psiquiátrica.

Conclusión: La reforma psiquiátrica debe ser una meta a ser seguida por los profesionales de la salud, sin embargo, no se pueden olvidar las necesidades específicas de los pacientes, tampoco desconsiderar los aspectos técnicos.

Palabras-clave: Dependencia química, reforma psiquiátrica, desinstitucionalización.

Título: Repercusiones del cierre de la Unidad de Desintoxicación del Hospital Psiquiátrico San Pedro

\section{Endereço para correspondência:}

Renata Brasil Araujo

Av. Venâncio Aires, 1092 ap.73

90040-192 - Porto Alegre - RS

Fone: (0xx51) 9965.9726

E-mail: renataudbrasil@hotmail.com

Copyright $\odot$ Revista de Psiquiatria

do Rio Grande do Sul - SPRS 